\title{
A!
}

This is an electronic reprint of the original article.

This reprint may differ from the original in pagination and typographic detail.

Marin Suarez, Marco; Peltonen, Joonas; Pekola, J.P.

\section{Active Quasiparticle Suppression in a Non-Equilibrium Superconductor}

Published in:

Nano Letters

DOI:

10.1021/acs.nanolett.0c01264

Published: 08/07/2020

Document Version

Publisher's PDF, also known as Version of record

Published under the following license:

CC BY

Please cite the original version:

Marin Suarez, M., Peltonen, J., \& Pekola, J. P. (2020). Active Quasiparticle Suppression in a Non-Equilibrium Superconductor. Nano Letters, 20(7), 5065-5071. https://doi.org/10.1021/acs.nanolett.0c01264

This material is protected by copyright and other intellectual property rights, and duplication or sale of all or part of any of the repository collections is not permitted, except that material may be duplicated by you for your research use or educational purposes in electronic or print form. You must obtain permission for any other use. Electronic or print copies may not be offered, whether for sale or otherwise to anyone who is not an authorised user. 


\title{
Active Quasiparticle Suppression in a Non-Equilibrium Superconductor
}

\author{
Marco Marín-Suárez,* Joonas T. Peltonen, and Jukka P. Pekola
}

Cite This: https://dx.doi.org/10.1021/acs.nanolett.0c01264

ABSTRACT: Quasiparticle (qp) poisoning is a major issue that impairs the operation of various superconducting devices. Even though these devices are often operated at temperatures well below the critical point where the number density of excitations is expected to be exponentially suppressed, their bare operation and stray microwave radiation excite the non-equilibrium qp's. Here we use voltage-biased superconducting junctions to demonstrate and quantify qp extraction in the turnstile operation of a superconductor-insulator-normal metal-insulator-superconductor single-electron transistor. In this operation regime, excitations are injected into the superconducting leads at a rate proportional to

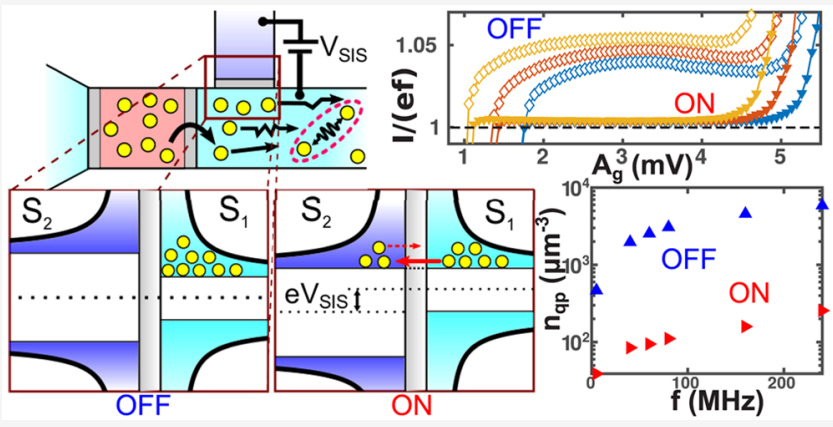
the driving frequency. We reach a reduction of density by an order of magnitude even for the highest injection rate of $2.4 \times 10^{8}$ qp's per second when extraction is turned on.

KEYWORDS: superconducting devices, quasiparticle poisoning, quasiparticle extraction, quasiparticle control, hybrid single-electron turnstile

$\mathrm{I}_{\mathrm{n}}^{\mathrm{n}}$ n superconducting circuits, it is important to minimize the number of non-equilibrium quasiparticles, as they deteriorate the operation of various devices, such as the coherence of quantum bits based on Josephson junctions ${ }^{1-5}$ or Majorana nanowires, ${ }^{6,7}$ cooling power of superconducting microcoolers, ${ }^{8,9}$ sensitivity of kinetic inductance detectors, ${ }^{10-12}$ and performance of superconducting resonators in other applications. ${ }^{13,14}$ In principle, bringing the system to temperatures $T$ much below the superconducting transition should reduce the number of excitations, as at $k_{\mathrm{B}} T \ll \Delta$ (here $k_{\mathrm{B}}$ is the Boltzmann constant and $\Delta$ is the superconducting energy gap) their equilibrium number density $n_{\mathrm{qp}}$ is suppressed exponentially. It has been demonstrated, however, that, when the devices are operated, quasiparticle (qp) excitations are created, caused typically by the drive signals or by stray microwave photons from hotter stages of the refrigerator, ${ }^{1,15-18}$ or by ionizing radiation. ${ }^{19}$ To overcome this "qp poisoning", several methods have been studied. These include introduction of normal metal traps, ${ }^{20-22}$ geometry optimization, ${ }^{15,23}$ vortex traps by magnetic field, ${ }^{13,23-25}$ gap engineering by variation of the film thickness, ${ }^{26-29}$ or phonon traps. ${ }^{30,31}$ Recently, blockage of qp's by a voltage-filter-tuned superconducting gap has been demonstrated, ${ }^{32}$ and employing a lower gap superconductor as a qp trap has been analyzed in detail. ${ }^{29}$ Although the crucial role of low qp density was identified already in early studies of superconducting qubits ${ }^{33-35}$ and related single-charge circuits, ${ }^{26,36}$ further understanding of the generation mechanisms and reducing $n_{\mathrm{qp}}$ remains the topic of an ever increasing intense research activity ${ }^{17,37-40}$ as the effort to increase the coherence times of superconducting qubits continues.

For radio frequency (rf) driven superconductor-insulatornormal metal-insulator-superconductor (SINIS) turnstiles, ${ }^{41}$ operated as a source of quantized electric current, ${ }^{42}$ both driveinduced and background qp's are the most severe limitation to reaching metrological current quantization accuracy. ${ }^{15,42}$ In this work, we show that such a hybrid single-electron transistor (SET, see Figure 1a for a typical sample) functions as a sensitive, practical, and quantitative detector of the qp density of its superconducting electrodes, that could be integrated with a variety of other mesoscopic superconducting devices to probe their $n_{\mathrm{qp}}$. Under rf drive, hybrid SETs present a turnstile for single electrons, a simple-to-operate candidate realization for a solid-state standard of electric current. ${ }^{41,43}$ For a wide range of parameters, the output current $I=$ ef is determined only by the drive frequency $f$ and electron charge $e$ (see Figure $1 \mathrm{~d}$ for typical measurements in this regime). Here the nonequilibrium state results from $\mathrm{qp}$ injection (Figure $1 \mathrm{~b}$ ) when the drive signal at frequency $f$ is applied to the gate electrode of the transistor. Earlier work ${ }^{15}$ demonstrated that the influence

Received: March 23, 2020

Revised: June 9, 2020 


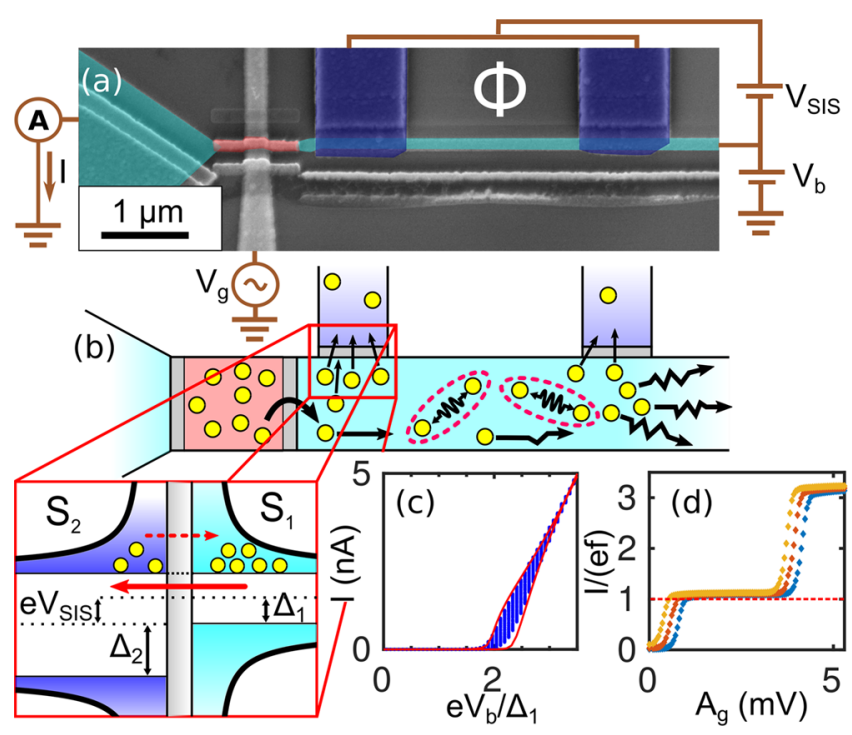

Figure 1. Qp extraction probed in a SINIS SET. (a) Electronic scheme for turnstile operation of the hybrid SET with an integrated $S_{1} I_{2}$ cooler, together with a false color scanning electron microscope image of sample A. Light red marks the normal metal and blue the superconducting parts, with light (dark) blue designating the lower (higher) gap superconductor. (b) Schematic sketch of the device. Quasiparticles are injected to the narrow lead where relaxation is inefficient. Qp excitations are extracted via biased $S_{1} I_{2}$ junctions, and recombination and scattering processes are still present along the lead. The inset shows that when the $S_{1} I S_{2}$ junction is biased at $e V_{\text {SIS }}=\mid \Delta_{1}$ $-\Delta_{2} \mid$ the singularities in the qp densities of states match and excitations are transferred from the lead with $\Delta_{1}$ to the reservoir with $\Delta_{2}$. (c) $I V$ curves of sample B in the positive bias regime, for a large number of different gate voltages. The red lines are simulations for the envelope curves, and the blue dots are the experimental data. (d) Measurements for sample A in the turnstile operation at $f=10 \mathrm{MHz}$ with $V_{\mathrm{b}}=120,160$, and $200 \mu \mathrm{V}$ as blue, red, and yellow symbols, respectively. Here the current is measured against the amplitude of the gate signal $A_{\mathrm{g}}$, and the red dashed line illustrates the ideal value of $I=e f$ for the first plateau.

of drive-induced and environmental qp's to a SINIS turnstile can be reduced by improving the geometry of the $S$ electrodes and by shielding from residual microwave radiation, respectively.

Here we combine the turnstile with an independent, in situ control-both extraction and injection-of qp's. The qp poisoning can be reduced by suitably voltage biasing superconductor-insulator-superconductor $\left(\mathrm{S}_{1} \mathrm{IS}_{2}\right)$ junctions with different superconducting gaps $\left(\Delta_{1}\right.$ and $\Delta_{2}$, respectively) where excitations are extracted from $S_{1}$ to $S_{2}$ as long as $\Delta_{1}<$ $\Delta_{2}{ }^{44-47}$ In an important experiment, this effect has been used to cool one of the leads of a single-Cooper pair transistor ${ }^{48}$ in a similar manner as refrigeration by normal metal-insulatorsuperconductor junctions ${ }^{49-51}$ has been used to cool down the normal lead. However, when using a single Cooper-pair transistor, it was not possible to control the mechanism or rate of $\mathrm{qp}$ creation, making it more difficult to quantify the population reduction due to the biased $S_{1} I_{2}$ junction. On the contrary, hybrid single-electron transistors allow for quantitative control. To that end, here we demonstrate that direct $S_{1} I_{2}$ cooling of the turnstile leads offers promise to fully extract the drive-induced qp's under typical pumping conditions, in particular when bulky or thick electrodes cannot be utilized.
The evacuation of qp's is manifested by the stabilization of current to ef in the SET turnstile operation. ${ }^{15}$ The qp extraction can be tuned by varying the voltage biasing of the $\mathrm{S}_{1} \mathrm{IS}_{2}$ junction. However, non-equilibrium qp's are also subjected to recombination and diffusion processes along the electrode, as depicted in panel $\mathrm{b}$ of Figure 1 . Due to the exponential dependence of $n_{\mathrm{qp}} \approx D\left(E_{\mathrm{F}}\right) \sqrt{2 \Delta k_{\mathrm{B}} T} \mathrm{e}^{-\Delta / k_{\mathrm{B}} T}$ on $\Delta$, the number of excitations is lower in the higher gap film. Here $D\left(E_{\mathrm{F}}\right)$ denotes the normal-state density of states at the Fermi energy and $T$ is the electronic temperature of the superconductor. Therefore, providing sufficient energy to qp's in the lower gap superconductor, biasing the junction such that the singularities in the superconducting densities of states align, will promote a transfer to the higher-gap superconductor (see the inset of Figure $1 \mathrm{~b}$ )..$^{52}$ Although two superconductors with different energy gaps are required, the qp extraction in a voltage-biased tunnel junction is in sharp contrast to those gap engineering methods ${ }^{26-29}$ where qp's are passively trapped in lower-gap regions away from the relevant operational zones of the device. To implement the qp evacuation and the probing of $n_{\mathrm{qp}}$ experimentally, we fabricate and measure a series of aluminum-based samples, cooled down in a dilution refrigerator reaching an electronic base temperature of $T_{\mathrm{b}} \approx$ $50 \mathrm{mK}$

Here we show detailed results for two devices for confirmation of results, both with copper as the turnstile normal-metal island, separated by aluminum-oxide barriers from two aluminum leads. The samples were patterned by electron-beam lithography, and metal deposition was done by multiangle shadow electron-beam evaporation; see the Supporting Information for further details on the fabrication process. One of the leads (left in Figure 1a) is made wide to trap qp's passively, while the other (right) is long and narrow, this way promoting an excess qp population: due to the geometry, qp diffusion away from the turnstile junction and their subsequent relaxation is intentionally poor in this narrow lead. ${ }^{15}$ The samples were fabricated using standard electronbeam lithography and shadow mask techniques. The narrow lead is the $S_{1}$ part of a $S_{1} I S_{2}$ Superconducting QUantum Interference Device (SQUID), whereas the fork-shaped electrode forms $\mathrm{S}_{2}$ with a higher superconducting gap. The different superconducting gaps are achieved by depositing a thicker aluminum layer as the turnstile lead $\left(d_{1} \approx 70 \mathrm{~nm}\right)$ and a thinner one for the rest of the SQUID $\left(d_{2} \approx 8 \mathrm{~nm}\right){ }^{53} \mathrm{~A}$ magnetic field perpendicular to the plane of the sample is applied to produce a flux $\Phi=\Phi_{0} / 2$ (here $\Phi_{0}=h /(2 e)$ is the magnetic flux quantum) in the SQUID loop and therefore suppress the supercurrent in the $\mathrm{S}_{1} \mathrm{IS}_{2}$ junctions so that subgap $V_{\text {SIS }}$ can be applied ${ }^{48}$ (see also the Supporting Information).

We characterize the samples by sweeping the gate voltage $V_{\mathrm{g}}$ between the "closed" $\left(n_{\mathrm{g}}=0\right)$ and "open" $\left(n_{\mathrm{g}}=0.5\right)$ states (with $n_{\mathrm{g}}=C_{\mathrm{g}} V_{\mathrm{g}} / e$, where $C_{\mathrm{g}}$ is the gate capacitance) and stepping the SET bias voltage $V_{\mathrm{b}}$. The $\mathrm{S}_{1} \mathrm{IS}_{2}$ junctions are first kept unbiased, named as the "cooler-off" case. The main parameters of the samples have been extracted from these measurements by fitting the measured current $I$ to currentvoltage curves calculated with a master equation approach taking into account sequential tunnelling and Andreev reflections $^{54}$ (see, for example, the Supporting Information and ref 55). One of these fits can be seen in Figure 1c. For sample A (B), the total normal-state tunnel resistance is $R_{\mathrm{T}}=$ $159.9 \mathrm{k} \Omega(63.0 \mathrm{k} \Omega)$, the charging energy of the island $E_{\mathrm{c}}=$ 
$0.95 \Delta_{1}\left(0.50 \Delta_{1}\right)$, the thickness of the $\mathrm{N}$ island $d=30 \mathrm{~nm}(40$ $\mathrm{nm})$, and the Dynes parameter ${ }^{18} \eta=1.0 \times 10^{-4}\left(7.5 \times 10^{-5}\right)$. For both samples, the superconducting energy gap $\Delta_{1}$ of the leads is $180 \mu \mathrm{eV}$, and the island's lateral dimensions $l \times w$ are 1 $\mu \mathrm{m} \times 100 \mathrm{~nm}$. See the Supporting Information for further details on the employed model where it is explicit that the island temperature is calculated according to power balance and that the area of a single conduction channel $\left(A_{\mathrm{ch}}\right)$ can be extracted from these data. However, it is more precise to extract $A_{\mathrm{ch}}$ from pumping measurements, since the effects of the second-order tunnelling are more pronounced in that operation regime. Since qp diffusion is altered by the different lead shapes, ${ }^{15}$ the DC fits were made by leaving the temperature of the long lead as a free parameter and assuming the base temperature of $50 \mathrm{mK}$ for the wide lead, estimating an electronic temperature of $\sim 150 \mathrm{mK}$ for the long lead. The values of $A_{\mathrm{ch}}, 9.5 \mathrm{~nm}^{2}\left(10 \mathrm{~nm}^{2}\right)$ for sample A (B), are lower by about a factor of 2 compared to previously reported values, ${ }^{56}$ yet the fits are relatively insensitive to the exact value of this parameter. We estimate these values from data with the cooler bias $V_{\text {SIS }}$ near a certain optimal point, where the Andreev effects are more noticeable, as will be seen later. By analyzing the differential conductance of the SQUID, it was possible to estimate $\Delta_{2}$ to be $\sim 235$ and $\sim 240 \pm 5 \mu \mathrm{eV}$ for samples A and $\mathrm{B}$, respectively.

The strong cooling by the superconducting junctions is evident in the turnstile operation of the SET shown in Figure 2. In this regime, a sinusoidal signal with an offset equivalent to $n_{\mathrm{g}}=0.5$ is applied to the gate electrode and the amplitude of this signal is swept while the SET current is measured. The presented data shows these measurements zoomed to the first current plateau (see Figure $1 \mathrm{~d}$ for a typical measurement in a
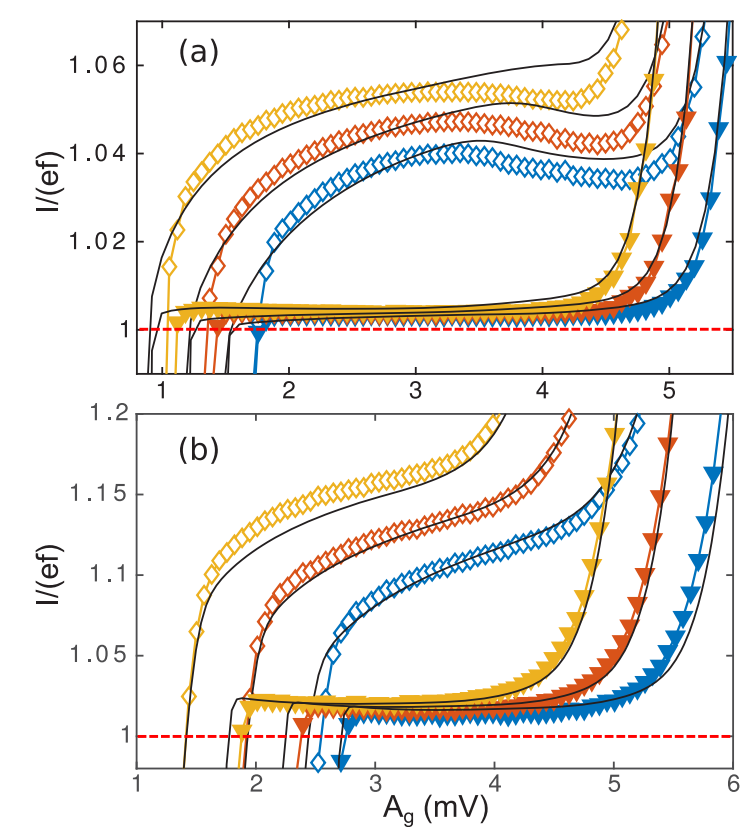

Figure 2. Improvement of the $I=$ ef pumping plateaus by the $\mathrm{S}_{1} \mathrm{IS}_{2}$ cooler. Data for the cooler-off (open diamonds) and cooler-on (filled triangles) cases compared with a model based on a master equation approach (solid black lines). Blue, red, and yellow curves correspond to $V_{\mathrm{b}}=120,160$, and $200 \mu \mathrm{V}$, respectively. (a) Sample A operating at $80 \mathrm{MHz}$; in the cooler-on case, the SQUID is biased at $60 \mu \mathrm{V}$. (b) Sample B operating at $100 \mathrm{MHz}$; here in the cooler-on cases, the SQUID is biased to $50 \mu \mathrm{V}$. wider range) for both samples. The plots display the behavior with the SQUID at zero bias and at a finite bias close to where optimum cooling is achieved. Besides displacing the plateau level from the expected value of $I=e f$, an elevated qp density in the leads close to the junctions makes the level depend on the SET bias voltage and hence the values of the current at the plateaus will spread. ${ }^{15}$ In the two cases of Figure 2, the "cooleron" $\left(V_{\text {SIS }} \neq 0\right)$ curves show a smaller deviation from the expected current than the cooler-off ones. Additionally, the bias dependence of the plateau level is weaker with the cooler active. All of this means that the density of excitations has been suppressed in the narrow lead by biasing the $S_{1} I_{2}$ junctions. For these particular devices, the remaining deviation is explained by the relatively low $E_{\mathrm{c}}<\Delta$ which promotes Andreev tunnelling, as well as by leakage current ${ }^{55}$ and remnant qp population as will be seen later.

To quantify the reduction of the qp density, the pumping data at the plateau shown in Figure 2 are compared with a model (black solid curves) based on a master equation approach similar to that in the DC case. In the model, the temperature of the island is varied at each amplitude value according to the proper power balance. Furthermore, since the used signal frequencies are high $\left(f>\tau_{\text {eff }}{ }^{-1}\right.$, with $\tau_{\text {eff }}$ being the effective qp relaxation time), it is suitable to model the system as if the temperature of the superconductors was constant throughout the operation cycle. ${ }^{16}$ Additionally, electronelectron relaxation is assumed to be fast enough to avoid branch imbalance. With these assumptions, the comparisons with the data are done with the temperature of the narrow lead as the only free parameter (device parameters are fixed by the DC measurements). As noted before, $n_{\mathrm{qp}}$ is related to the effective electron temperature $T$ in the superconducting lead obtained by means of the thermal balance model. These simulations allow one to deduce that the biasing of the $S_{1} I S_{2}$ junctions effectively cools the narrow long lead. Furthermore, the use of the pumping operation of a hybrid SET as a sensitive detector of qp's is justified by these calculations. Figure 3a shows a specific example of sample B biased at $V_{\mathrm{b}}=100 \mu \mathrm{V}$ and gate modulated with an amplitude of around $A_{\mathrm{g}}=3.5 \mathrm{mV}$. There it is evident that the plateau level grows with the excitation density in the lead. The behavior is nearly linear at high population but flattens for low values.

The behavior of the qp density when the cooler bias changes is analyzed by performing measurements where $V_{\text {SIS }}$ is swept while the gate drive amplitude $A_{\mathrm{g}}$ is fixed to approximately the middle of the first current plateau. Examples of such measurements are shown in Figure $3 \mathrm{~b}$ for device $\mathrm{A}$ and in panels $\mathrm{c}$ and $\mathrm{d}$ for device $\mathrm{B}$. The plateau level and the spreading of them for different bias values of the SET decrease until we reach $e V_{\text {SIS }} \approx \pm\left|\Delta_{2}-\Delta_{1}\right|^{57}$ (indicated by a dashed line in panels $b-d)$. At this point, the temperature of the lead is at a minimum and it changes only weakly until a threshold at which it starts to grow rapidly. Therefore, we can assert that when the cooler bias voltage reaches the optimum point the excitation population in the lead is at a minimum and at higher biases it starts to grow up first at a low rate and then rapidly when there are peaks in subgap current of the $S_{1} I S_{2}$ SQUID (see the Supporting Information for further details). Similarly, biasing at $V_{\text {SIS }}<0$ affects the system in the same way but this time by extracting hole-like excitations (consider the inset in Figure $1 \mathrm{~b}$ with the lower singularities aligned). We can also understand this by looking at the modeled cooling power of 


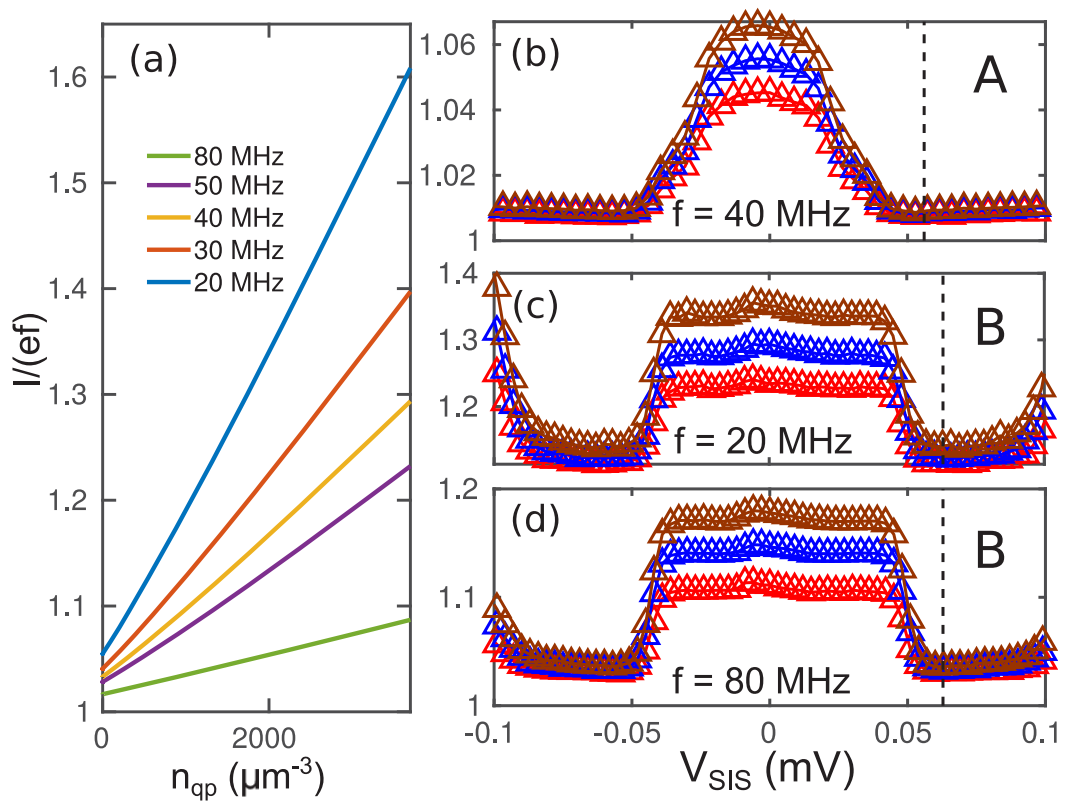

Figure 3. Sensitivity of current plateau to qp's. (a) Calculated curves showing how the current at the plateau varies with the qp density. The parameters of sample B were used in these calculations along with a bias of $V_{\mathrm{b}}=100 \mu \mathrm{V}$ and a gate amplitude of $A_{\mathrm{g}}=3.5 \mathrm{mV}$. (b) Measured current at the plateau as a function of the bias applied to the $S_{1} I_{2}$ cooler for device $\mathrm{A}$ at $f=40 \mathrm{MHz}$. From bottom to top, the curves correspond to $V_{\mathrm{b}}=$ 120,160 , and $200 \mu \mathrm{V}$, respectively. (c) As in part b for B at $20 \mathrm{MHz}$. (d) As in part c for $80 \mathrm{MHz}$. Dashed black lines indicate the optimal biasing point of the cooler.

the $S_{1} I_{2}$ junction ${ }^{58}$ (see the Supporting Information for details) when it begins to grow with $V_{\text {SIS }}$ toward a sharp peak, where the lead temperature is minimum, and then it decreases dramatically, corresponding to the zone with finite but less efficient cooling. Finally, at $e V_{\text {SIS }} \approx \Delta_{2}+\Delta_{1}$, qp's are injected into the lead with the smaller gap. No sharp dip is observed in the measured $I$ vs $V_{\text {SIS }}$ curves at $e V_{\text {SIS }}=\left|\Delta_{2}-\Delta_{1}\right|$, similar to earlier works on $S_{1} I_{2}$ cooling. ${ }^{47,48}$ The singularity matching peak in the cooling power is likely washed out due to lowfrequency noise in the $S_{1} I S_{2}$ cooler bias voltage, finite subgap density of states, and local inhomogeneities in the superconducting gap.

The influence of the $S_{1} I S_{2}$ cooler is qualitatively the same for both samples and independent of the operation frequency as well as of the SET bias, although the plateau levels are different; see the Supporting Information for additional measurements to corroborate this fact. Note that this level approaches the ideal value but never reaches it even in the calculations for samples with low $E_{c}<\Delta$ (see Figure 3a). In these devices with low $E_{\mathcal{c}}$, we observe a clear crossover from excess qp-dominated to Andreev-reflection-limited current quantization as the cooler is turned on.

We estimate the lead temperature for a wide range of operation frequencies. Figures $4 a$ and $b$ show that the lead temperature $T$ extracted from the fitting procedure and in turn $n_{\mathrm{qp}}$ (Figures $4 \mathrm{c}$ and d) grow with increasing frequency also in the cooler-on case. However, in sample B, the curve for the cooler-on case flattens at $f>40 \mathrm{MHz}$, since the SET behavior loses sensitivity to qp density in this operation regime (see Figure $3 \mathrm{a})$. For the near optimal cooler bias $\left(V_{\mathrm{SIS}} \approx 50\right.$ and 60 $\mu \mathrm{V}$ for samples $\mathrm{A}$ and $\mathrm{B}$, respectively), the dependence of $n_{\mathrm{qp}}$ versus the operation frequency is resemblant. This comes from the fact that qp transport is dominated by diffusion. There is a dramatic drop in the qp density due to the biasing of the $S_{1} I_{2}$ junctions. By linearly extrapolating the densities to the $f=0$ limit, it is possible to see that there is a reduction between the

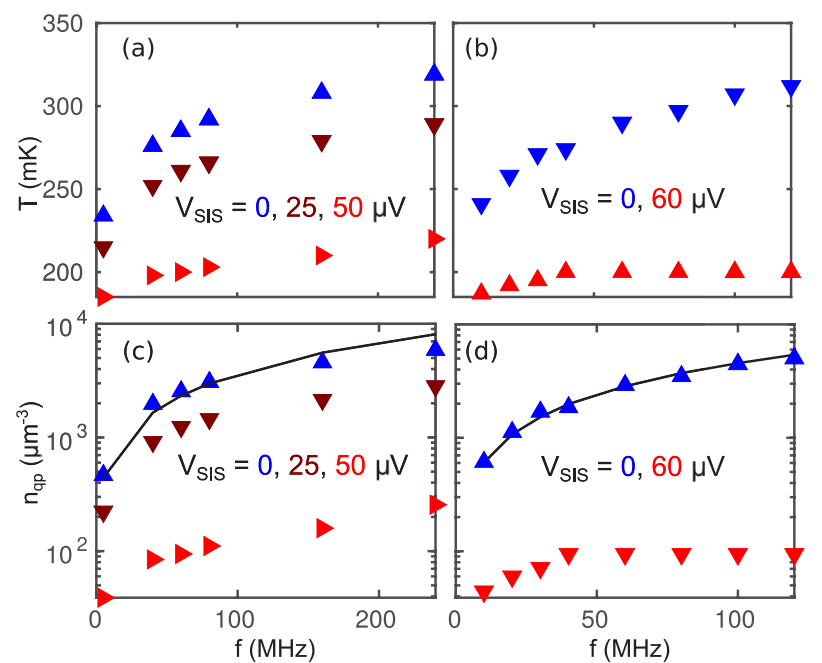

Figure 4. Qp density variation as a function of drive frequency. Estimated narrow lead temperature during the turnstile operation as a function of the gate signal frequency (a) for sample A and (b) for sample B. Estimated qp density at the junction as a function of the pumping frequency (c) for sample A and (d) for sample B, corresponding to the data in panels $a$ and $b$, respectively. The black solid lines are fittings to eq 1 .

"off" and "on" case by an order of magnitude also in this situation. We conclude that the cooler suppresses also the ever present excess qp population $n_{\mathrm{qp}, 0}$, generated by a nonequilibrium environment, ${ }^{59-61}$ which in turn should diminish the subgap current in the DC regime. On the other hand, it is seen that the biased $S_{1} I S_{2}$ junctions cannot totally remove this excess qp population in the leads. We expect that even more efficient evacuation can be achieved by placing the $S_{1} I_{2}$ junction closer to the injection junction-or even under itsince the highest concentration of qp's is near this point, ${ }^{21,51}$ 
and further diffusion, recombination, and phonon pair breaking, among other phenomena, can thus be avoided.

We measured further reference samples with identical aluminum lead geometry but without $S_{1} I_{2}$ SQUIDs and obtained the qp densities in them (for results, see the Supporting Information). The qp densities are similar to those shown in Figure 4 for the cooler-off case, and thus, there is no significant influence of the unbiased cooler junctions or the presence of their biasing circuit on the relaxation of the nonequilibrium excitations. Hence, the presented cooler-off densities should correspond approximately to densities in the case if the $S_{1} I S_{2}$ junctions were not present. Thus, the diffusion model in ref 15 , which is based on heat conduction, without considering normal metal traps, should hold. Within this model, the qp density is given by

$$
n_{\mathrm{qp}}=e^{2} D\left(E_{\mathrm{F}}\right) P_{\mathrm{inj}} \frac{\rho_{\mathrm{n}} l}{\varpi \vartheta} \sqrt{\frac{\pi}{2 k_{\mathrm{B}} T \Delta_{1}}}+n_{\mathrm{qp}, 0}
$$

where $l, \varpi$, and $\vartheta$ are the dimensions of the lead $(25 \mu \mathrm{m} \times 100$ $\mathrm{nm} \times 70 \mathrm{~nm})$ and $P_{\text {inj }}$ is the injected power which can be approximated by $\Delta_{1} f$ in the driving regime of the experiment. ${ }^{15}$ In the Supporting Information, we show that this is a good approximation. Notably, even at $f=100 \mathrm{MHz}$, the injected power for an aluminum-based device with $\Delta_{1}=200 \mu \mathrm{eV}$ is only around $3.2 \mathrm{fW}$, extractable by a submicron $S_{1} \mathrm{IS}_{2}$ junction. In addition, $\rho_{\mathrm{n}}$ is the aluminum normal state resistivity. Based on measurements of a number of separate test structures, we estimate $\rho_{\mathrm{n}} \approx 31 \Omega \mathrm{nm}$ at $77 \mathrm{~K}$ (see the Supporting Information for details). A few of these four-probe structures were cooled down to $4.2 \mathrm{~K}$ where we estimate a further $10 \%$ decrease of $\rho_{\mathrm{n}}$ below its $77 \mathrm{~K}$ value. The measured qp numbers are larger than those predicted by this model with no free parameters. Fitting eq 1 to the data using $\rho_{\mathrm{n}}$ as a free parameter yields $\rho_{\mathrm{n}} \sim 90 \Omega \mathrm{nm}$. These fits are shown as black lines in panels $\mathrm{c}$ and $\mathrm{d}$ of Figure 4. Further experiments are needed to understand the discrepancy, observed also in ref 62 for devices with higher charging energy.

At $f=0$ and $V_{\text {SIS }}=0$, we estimate $n_{\mathrm{qp}, 0} \approx 250 \mu \mathrm{m}^{-3}$ for both samples, obtained from fits to dc $I V$ characteristics of the turnstile. These background qp densities for the cooler-off case are roughly 2 orders of magnitude higher than those observed for highly shielded SINIS turnstiles, ${ }^{15}$ or superconducting qubits and resonators. ${ }^{11,37,63}$ The excess in $n_{\mathrm{qp}, 0}$ compared to ref 15 originates from the lack of a microwave-tight indium seal in the sample holders employed in these measurements and from the lack of any normal metal traps and restricted geometry-narrow, thin, long- of the $S$ electrode with the $\mathrm{S}_{1} \mathrm{IS}_{2}$ junctions. To bring down $n_{\mathrm{qp}, 0}$, a sample holder with a higher level of shielding can be utilized, and the $S_{2}$ electrode can be fabricated with separate lithography and deposition steps that do not limit the junction geometry as with the multiangle shadow evaporation technique where both NIS and $\mathrm{S}_{1} \mathrm{IS}_{2}$ junctions are created with a single mask.

The applicability of the qp extraction and detection techniques considered in this work extends beyond SINIS turnstiles and improving the accuracy of their pumped current. First, one can envision straightforward integration of $S_{1} I_{2}$ coolers, for instance, with absorbers of kinetic inductance detectors, or into superconducting resonators analogously to NIS coolers. ${ }^{64}$ When the bottom electrodes of the junctions are fabricated in a separate lithography and deposition step, their area and geometry can be adjusted at will. Furthermore, instead of tuning the gap by the film thickness, different superconducting materials can be utilized. Second, the SINIS turnstile, demonstrated here to function as a sensitive and direct qp probe, can be combined with various superconducting devices to measure the background $\mathrm{qp}$ density. This high-impedance, non-invasive probe can test the level of microwave shielding also in setups with sensitive nonsuperconducting devices. Finally, we have shown the driven hybrid turnstile to act as a highly controlled qp injector that could be used to investigate the qp sensitivity and qp trap efficiency of superconducting qubits and other devices.

In summary, we have been able to demonstrate active extraction of non-equilibrium excitations from a superconductor. A more than 1 order of magnitude reduction from values as high as $5.8 \times 10^{3}$ to $260 \mu \mathrm{m}^{-3}$ at the highest studied injection rate of $2.4 \times 10^{8} \mathrm{~s}^{-1}$ was achieved. Furthermore, in the limit of no qp injection $(f \rightarrow 0)$, we find a similar reduction, although a finite population of environmentally created excitations remains. Our work shows that the qp density in the superconducting electrodes can be controlled by active qp traps, here demonstrated for the first time with in situ control of qp injection.

\section{ASSOCIATED CONTENT}

\section{SI Supporting Information}

The Supporting Information is available free of charge at https://pubs.acs.org/doi/10.1021/acs.nanolett.0c01264.

Calculations of the cooling power of $S_{1} I_{2}$ junctions are contained here, as well as a model for the power transfer in a driven system. Additionally, details on fabrication and measurement procedures are given. Further details on the theoretical background and numerical calculations of SINIS SETs as well as results for additional and reference measurements are provided. Finally, details on the aluminum resistivity are given. (PDF)

\section{AUTHOR INFORMATION}

\section{Corresponding Author}

Marco Marín-Suárez - Pico group, QTF Centre of Excellence, Department of Applied Physics, Aalto University, FI-000 76 Aalto, Finland; @ orcid.org/0000-0003-2057-7707; Email: marco.marinsuarez@aalto.fi

\section{Authors}

Joonas T. Peltonen - Pico group, QTF Centre of Excellence, Department of Applied Physics, Aalto University, FI-000 76 Aalto, Finland; 10 orcid.org/0000-0002-3903-7623

Jukka P. Pekola - Pico group, QTF Centre of Excellence, Department of Applied Physics, Aalto University, FI-000 76 Aalto, Finland

Complete contact information is available at: https://pubs.acs.org/10.1021/acs.nanolett.0c01264

\section{Author Contributions}

M.M.-S. performed simulations, analyzed the data, and performed the resistivity measurements. J.T.P. fabricated the devices and performed the rest of the experiments. The calculation of the injected power was done by J.P.P and M.M.S. The manuscript was written by M.M.-S. with important input from J.T.P. and J.P.P. M.M.-S. and J.T.P. contributed equally to the research. 


\section{Notes}

The authors declare no competing financial interest.

\section{ACKNOWLEDGMENTS}

We acknowledge E. T. Mannila, D. Golubev, B. Karimi, V. F. Maisi, and I. M. Khaymovich for useful discussions. This research made use of Otaniemi Research Infrastructure for Micro and Nanotechnologies (OtaNano) and its Low Temperature Laboratory, which is part of the European Microkelvin Platform (EMP). This work is funded through Academy of Finland Grant Nos. 297240, 312057, and 303677.

\section{REFERENCES}

(1) Martinis, J. M.; Ansmann, M.; Aumentado, J. Energy decay in superconducting Josephson-junction qubits from nonequilibrium quasiparticle excitations. Phys. Rev. Lett. 2009, 103, 097002.

(2) Paik, H.; Schuster, D. I.; Bishop, L. S.; Kirchmair, G.; Catelani, G.; Sears, A. P.; Johnson, B. R.; Reagor, M. J.; Frunzio, L.; Glazman, L. I.; Girvin, S. M.; Devoret, M. H.; Schoelkopf, R. J. Observation of high coherence in Josephson junction qubits measured in a threedimensional circuit QED architecture. Phys. Rev. Lett. 2011, 107, 240501.

(3) Barends, R.; Wenner, J.; Lenander, M.; Chen, Y.; Bialczak, R. C.; Kelly, J.; Lucero, E.; O’Malley, P.; Mariantoni, M.; Sank, D.; Wang, H.; White, T. C.; Yin, Y.; Zhao, J.; Cleland, A. N.; Martinis, J. M.; Baselmans, J. J. A. Minimizing quasiparticle generation from stray infrared light in superconducting quantum circuits. Appl. Phys. Lett. 2011, 99, 113507.

(4) Catelani, G.; Koch, J.; Frunzio, L.; Schoelkopf, R. J.; Devoret, M. H.; Glazman, L. I. Quasiparticle relaxation of superconducting qubits in the presence of flux. Phys. Rev. Lett. 2011, 106, 077002.

(5) Lenander, M.; Wang, H.; Bialczak, R. C.; Lucero, E.; Mariantoni, M.; Neeley, M.; O’Connell, A. D.; Sank, D.; Weides, M.; Wenner, J.; Yamamoto, T.; Yin, Y.; Zhao, J.; Cleland, A. N.; Martinis, J. M. Measurement of energy decay in superconducting qubits from nonequilibrium quasiparticles. Phys. Rev. B: Condens. Matter Mater. Phys. 2011, 84, 024501.

(6) Rainis, D.; Loss, D. Majorana qubit decoherence by quasiparticle poisoning. Phys. Rev. B: Condens. Matter Mater. Phys. 2012, 85, 174533.

(7) Higginbotham, A. P.; Albrecht, S. M.; Kiršanskas, G.; Chang, W.; Kuemmeth, F.; Krogstrup, P.; Jespersen, T. S.; Nygård, J.; Flensberg, K.; Marcus, C. M. Parity lifetime of bound states in a proximitized semiconductor nanowire. Nat. Phys. 2015, 11, 1017.

(8) Muhonen, J. T.; Meschke, M.; Pekola, J. P. Micrometre-scale refrigerators. Rep. Prog. Phys. 2012, 75, 046501.

(9) Nguyen, H. Q.; Aref, T.; Kauppila, V. J.; Meschke, M.; Winkelmann, C. B.; Courtois, H.; Pekola, J. P. Trapping hot quasiparticles in a high-power superconducting electronic cooler. New J. Phys. 2013, 15, 085013.

(10) de Visser, P. J.; Baselmans, J. J. A.; Diener, P.; Yates, S. J. C.; Endo, A.; Klapwijk, T. M. Number fluctuations of sparse quasiparticles in a superconductor. Phys. Rev. Lett. 2011, 106, 167004. (11) de Visser, P. J.; Baselmans, J. J. A.; Diener, P.; Yates, S. J. C.; Endo, A.; Klapwijk, T. M. Generation-recombination noise: The fundamental sensitivity limit for kinetic inductance detectors. J. Low Temp. Phys. 2012, 167, 335.

(12) Patel, U.; Pechenezhskiy, I. V.; Plourde, B. L. T.; Vavilov, M. G.; McDermott, R. Phonon-mediated quasiparticle poisoning of superconducting microwave resonators. Phys. Rev. B: Condens. Matter Mater. Phys. 2017, 96, 220501.

(13) Nsanzineza, I.; Plourde, B. L. T. Trapping a single vortex and reducing quasiparticles in a superconducting resonator. Phys. Rev. Lett. 2014, 113, 117002 .

(14) Grünhaupt, L.; Maleeva, N.; Skacel, S. T.; Calvo, M.; LevyBertrand, F.; Ustinov, A. V.; Rotzinger, H.; Monfardini, A.; Catelani, G.; Pop, I. M. Loss mechanisms and quasiparticle dynamics in superconducting microwave resonators made of thin-film granular aluminum. Phys. Rev. Lett. 2018, 121, 117001.

(15) Knowles, H. S.; Maisi, V. F.; Pekola, J. P. Probing quasiparticle excitations in a hybrid single electron transistor. Appl. Phys. Lett. 2012, 100, 262601.

(16) Maisi, V. F.; Lotkhov, S. V.; Kemppinen, A.; Heimes, A.; Muhonen, J. T.; Pekola, J. P. Excitation of single quasiparticles in a small superconducting $\mathrm{Al}$ island connected to normal-metal leads by tunnel junctions. Phys. Rev. Lett. 2013, 111, 147001.

(17) Gustavsson, S.; Yan, F.; Catelani, G.; Bylander, J.; Kamal, A.; Birenbaum, J.; Hover, D.; Rosenberg, D.; Samach, G.; Sears, A. P.; Weber, S. J.; Yoder, J. L.; Clarke, J.; Kerman, A. J.; Yoshihara, F.; Nakamura, Y.; Orlando, T. P.; Oliver, W. D. Suppressing relaxation in superconducting qubits by quasiparticle pumping. Science 2016, 354, 1573.

(18) Saira, O.-P.; Möttönen, M.; Maisi, V. F.; Pekola, J. P. Environmentally activated tunneling events in a hybrid single-electron box. Phys. Rev. B: Condens. Matter Mater. Phys. 2010, 82, 155443.

(19) Vepsäläinen, A.; Karamlou, A. H.; Orrell, J. L.; Dogra, A. S.; Loer, B.; Vasconcelos, F.; Kim, D. K.; Melville, A. J.; Niedzielski, B. M.; Yoder, J. L.; Gustavsson, S.; Formaggio, J. A.; VanDevender, B. A.; Oliver, W. D. Impact of ionizing radiation on superconducting qubit coherence. 2020, arXiv:2001.09190. arXiv.org e-Print archive. https://arxiv.org/abs/arXiv:2001.09190 (accessed April 23, 2020).

(20) Goldie, D. J.; Booth, N. E.; Patel, C.; Salmon, G. L. Quasiparticle trapping from a single-crystal superconductor into a normal-metal film via the proximity effect. Phys. Rev. Lett. 1990, 64, 954.

(21) Riwar, R.-P.; Hosseinkhani, A.; Burkhart, L. D.; Gao, Y. Y.; Schoelkopf, R. J.; Glazman, L. I.; Catelani, G. Normal-metal quasiparticle traps for superconducting qubits. Phys. Rev. B: Condens. Matter Mater. Phys. 2016, 94, 104516.

(22) Hosseinkhani, A.; Catelani, G. Proximity effect in normal-metal quasiparticle traps. Phys. Rev. B: Condens. Matter Mater. Phys. 2018, 97, 054513.

(23) Wang, C.; Gao, Y. Y.; Pop, I. M.; Vool, U.; Axline, C.; Brecht, T.; Heeres, R. W.; Frunzio, L.; Devoret, M. H.; Catelani, G.; Glazman, L.; Schoelkopf, R. J. Measurement and control of quasiparticle dynamics in a superconducting qubit. Nat. Commun. 2014, 5, 5836.

(24) Vool, U.; Pop, I. M.; Sliwa, K.; Abdo, B.; Wang, C.; Brecht, T.; Gao, Y. Y.; Shankar, S.; Hatridge, M.; Catelani, G.; Mirrahimi, M.; Frunzio, L.; Schoelkopf, R. J.; Glazman, L. I.; Devoret, M. H. Nonpoissonian quantum jumps of a fluxonium qubit due to quasiparticle excitations. Phys. Rev. Lett. 2014, 113, 247001.

(25) Taupin, M.; Khaymovich, I. M.; Meschke, M.; Mel'nikov, A. S.; Pekola, J. P. Tunable quasiparticle trapping in Meissner and vortex states of mesoscopic superconductors. Nat. Commun. 2016, 7, 10977.

(26) Aumentado, J.; Keller, M. W.; Martinis, J. M.; Devoret, M. H. Nonequilibrium quasiparticles and $2 e$ periodicity in single-Cooperpair transistors. Phys. Rev. Lett. 2004, 92, 066802.

(27) Yamamoto, T.; Nakamura, Y.; Pashkin, Y. A.; Astafiev, O.; Tsai, J. S. Parity effect in superconducting aluminum single electron transistors with spatial gap profile controlled by film thickness. Appl. Phys. Lett. 2006, 88, 212509.

(28) Sun, L.; DiCarlo, L.; Reed, M. D.; Catelani, G.; Bishop, L. S.; Schuster, D. I.; Johnson, B. R.; Yang, G. A.; Frunzio, L.; Glazman, L.; Devoret, M. H.; Schoelkopf, R. J. Measurements of quasiparticle tunneling dynamics in a band-gap-engineered transmon qubit. Phys. Rev. Lett. 2012, 108, 230509.

(29) Riwar, R.-P.; Catelani, G. Efficient quasiparticle traps with low dissipation through gap engineering. Phys. Rev. B: Condens. Matter Mater. Phys. 2019, 100, 144514.

(30) Henriques, F.; Valenti, F.; Charpentier, T.; Lagoin, M.; Gouriou, C.; Martínez, M.; Cardani, L.; Vignati, M.; Gränhaupt, L.; Gusenkova, D.; Ferrero, J.; Skacel, S. T.; Wernsdorfer, W.; Ustinov, A. V.; Catelani, G.; Sander, O.; Pop, I. M. Phonon traps reduce the quasiparticle density in superconducting circuits. Appl. Phys. Lett. 2019, 115, 212601. 
(31) Karatsu, K.; Endo, A.; Bueno, J.; de Visser, P. J.; Barends, R.; Thoen, D. J.; Murugesan, V.; Tomita, N.; Baselmans, J. J. A. Mitigation of cosmic ray effect on microwave kinetic inductance detector arrays. Appl. Phys. Lett. 2019, 114, 032601.

(32) Ménard, G. C.; Malinowski, F. K.; Puglia, D.; Pikulin, D. I.; Karzig, T.; Bauer, B.; Krogstrup, P.; Marcus, C. M. Suppressing quasiparticle poisoning with a voltage-controlled filter. Phys. Rev. B: Condens. Matter Mater. Phys. 2019, 100, 165307.

(33) Lang, K.; Nam, S.; Aumentado, J.; Urbina, C.; Martinis, J. Banishing quasiparticles from Josephson-junction qubits: Why and how to do it. IEEE Trans. Appl. Supercond. 2003, 13, 989.

(34) Lutchyn, R.; Glazman, L.; Larkin, A. Quasiparticle decay rate of Josephson charge qubit oscillations. Phys. Rev. B: Condens. Matter Mater. Phys. 2005, 72, 014517.

(35) Shaw, M. D.; Lutchyn, R. M.; Delsing, P.; Echternach, P. M. Kinetics of nonequilibrium quasiparticle tunneling in superconducting charge qubits. Phys. Rev. B: Condens. Matter Mater. Phys. 2008, 78, 024503.

(36) Männik, J.; Lukens, J. E. Effect of measurement on the periodicity of the Coulomb staircase of a superconducting box. Phys. Rev. Lett. 2004, 92, 057004.

(37) Ristè, D.; Bultink, C. C.; Tiggelman, M. J.; Schouten, R. N.; Lehnert, K. W.; DiCarlo, L. Millisecond charge-parity fluctuations and induced decoherence in a superconducting transmon qubit. Nat. Commun. 2013, 4, 1913.

(38) Pop, I. M.; Geerlings, K.; Catelani, G.; Schoelkopf, R. J.; Glazman, L. I.; Devoret, M. H. Coherent suppression of electromagnetic dissipation due to superconducting quasiparticles. Nature 2014, 508, 369.

(39) Serniak, K.; Hays, M.; de Lange, G.; Diamond, S.; Shankar, S.; Burkhart, L. D.; Frunzio, L.; Houzet, M.; Devoret, M. H. Hot nonequilibrium quasiparticles in transmon qubits. Phys. Rev. Lett. 2018, 121, 157701.

(40) Serniak, K.; Diamond, S.; Hays, M.; Fatemi, V.; Shankar, S.; Frunzio, L.; Schoelkopf, R.; Devoret, M. Direct dispersive monitoring of charge parity in offset-charge-sensitive transmons. Phys. Rev. Appl. 2019, 12, 014052.

(41) Pekola, J. P.; Vartiainen, J. J.; Möttönen, M.; Saira, O.-P.; Meschke, M.; Averin, D. V. Hybrid single-electron transistor as a source of quantized electric current. Nat. Phys. 2008, 4, 120.

(42) Pekola, J. P.; Saira, O.-P.; Maisi, V. F.; Kemppinen, A.; Möttönen, M.; Pashkin, Y. A.; Averin, D. V. Single-electron current sources: Toward a refined definition of the ampere. Rev. Mod. Phys. 2013, 85, 1421.

(43) Maisi, V. F.; Pashkin, Y. A.; Kafanov, S.; Tsai, J.-S.; Pekola, J. P. Parallel pumping of electrons. New J. Phys. 2009, 11, 113057.

(44) Chi, C. C.; Clarke, J. Enhancement of the energy gap in superconducting aluminum by tunneling extraction of quasiparticles. Phys. Rev. B: Condens. Matter Mater. Phys. 1979, 20, 4465.

(45) Blamire, M. G.; Kirk, E. C. G.; Evetts, J. E.; Klapwijk, T. M. Extreme critical-temperature enhancement of Al by tunneling in $\mathrm{Nb}$ / $\mathrm{AlO}_{x} / \mathrm{Al} / \mathrm{AlO}_{x} / \mathrm{Nb}$ tunnel junctions. Phys. Rev. Lett. 1991, 66, 220.

(46) Heslinga, D. R.; Klapwijk, T. M. Enhancement of superconductivity far above the critical temperature in double-barrier tunnel junctions. Phys. Rev. B: Condens. Matter Mater. Phys. 1993, 47, 5157.

(47) Manninen, A. J.; Suoknuuti, J. K.; Leivo, M. M.; Pekola, J. P. Cooling of a superconductor by quasiparticle tunneling. Appl. Phys. Lett. 1999, 74, 3020.

(48) Ferguson, A. J. Quasiparticle cooling of a single Cooper pair transistor. Appl. Phys. Lett. 2008, 93, 052501.

(49) Pekola, J. P.; Anghel, D. V.; Suppula, T. I.; Suoknuuti, J. K.; Manninen, A. J.; Manninen, M. Trapping of quasiparticles of a nonequilibrium superconductor. Appl. Phys. Lett. 2000, 76, 2782.

(50) Giazotto, F.; Heikkilä, T. T.; Luukanen, A.; Savin, A. M.; Pekola, J. P. Opportunities for mesoscopics in thermometry and refrigeration: Physics and applications. Rev. Mod. Phys. 2006, 78, 217.
(51) Rajauria, S.; Courtois, H.; Pannetier, B. Quasiparticle-diffusionbased heating in superconductor tunneling microcoolers. Phys. Rev. B: Condens. Matter Mater. Phys. 2009, 80, 214521.

(52) Parmenter, R. H. Enhancement of superconductivity by extraction of normal carriers. Phys. Rev. Lett. 1961, 7, 274.

(53) Court, N. A.; Ferguson, A. J.; Clark, R. G. Energy gap measurement of nanostructured aluminium thin films for single Cooper-pair devices. Supercond. Sci. Technol. 2008, 21, 015013.

(54) Hekking, F. W. J.; Nazarov, Y. V. Subgap conductivity of a superconductor-normal-metal tunnel interface. Phys. Rev. B: Condens. Matter Mater. Phys. 1994, 49, 6847.

(55) Aref, T.; Maisi, V. F.; Gustafsson, M. V.; Delsing, P.; Pekola, J. P. Andreev tunneling in charge pumping with SINIS turnstiles. EPL (Europhysics Letters) 2011, 96, 37008.

(56) Maisi, V. F.; Saira, O.-P.; Pashkin, Y. A.; Tsai, J. S.; Averin, D. V.; Pekola, J. P. Real-time observation of discrete Andreev tunneling events. Phys. Rev. Lett. 2011, 106, 217003.

(57) Melton, R. G.; Paterson, J. L.; Kaplan, S. B. Superconducting tunnel-junction refrigerator. Phys. Rev. B: Condens. Matter Mater. Phys. 1980, 21, 1858.

(58) Golubev, D.; Faivre, T.; Pekola, J. P. Heat transport through a Josephson junction. Phys. Rev. B: Condens. Matter Mater. Phys. 2013, $87,094522$.

(59) Lambert, N. J.; Edwards, M.; Esmail, A. A.; Pollock, F. A.; Barrett, S. D.; Lovett, B. W.; Ferguson, A. J. Experimental observation of the breaking and recombination of single Cooper pairs. Phys. Rev. B: Condens. Matter Mater. Phys. 2014, 90, 140503.

(60) Lambert, N. J.; Esmail, A. A.; Pollock, F. A.; Edwards, M.; Lovett, B. W.; Ferguson, A. J. Microwave irradiation and quasiparticles in a superconducting double dot. Phys. Rev. B: Condens. Matter Mater. Phys. 2017, 95, 235413.

(61) Rossignol, B.; Kloss, T.; Waintal, X. Role of quasiparticles in an electric circuit with Josephson junctions. Phys. Rev. Lett. 2019, 122, 207702.

(62) Mykkänen, E. From quantum metrology to applications; Kvanttimittatekniikasta sovelluksiin. Ph.D. thesis [Online], Aalto University, Espoo, Finland, October 24, 2018. http://urn.fi/ URN:ISBN:978-952-60-8276-9 (accessed on December 4, 2019).

(63) de Visser, P. J.; Baselmans, J. J. A.; Yates, S. J. C.; Diener, P.; Endo, A.; Klapwijk, T. M. Microwave-induced excess quasiparticles in superconducting resonators measured through correlated conductivity fluctuations. Appl. Phys. Lett. 2012, 100, 162601.

(64) Tan, K. Y.; Partanen, M.; Lake, R. E.; Govenius, J.; Masuda, S.; Möttönen, M. Quantum-circuit refrigerator. Nat. Commun. 2017, 8, 15189. 